\title{
SIMULATION BASED MODELING FOR A CYBERSECURE POWER GRID
}

\author{
Michael Mesham \\ Drexel University \\ 3141 Chestnut Street \\ Philadelphia, PA, USA \\ mm5243@drexel.edu
}

\author{
Mahmoud Fahmy \\ University of Miami \\ 1320 South Dixie Highway \\ Coral Gables, FL, USA \\ mxf898@miami.edu
}

\author{
Nurcin Celik \\ University of Miami \\ 1251 Memorial Drive \\ Coral Gables, FL, USA \\ celik@miami.edu
}

ABSTRACT

The North American bulk power system is one of the most vital infrastructures in modern society as it accounts for virtually all the electricity supplied to the United States, Canada, and a portion of Baja Notre California, Mexico. Cyberattacks, of all forms, are becoming increasingly prominent within power networks and other infrastructures whereas their resolution can consume a significant deal of time and monetary resources. This can be further worsened if there are subsequent physical attacks in the wake of a cyberattack, as the system downtime leaves the government, military, and other critical infrastructures incredibly vulnerable This research aims to investigate if different patterns of cyberattacks could be identified with speed using simulation and machine learning algorithms. More specifically, we design a simulation model that can help better defend against cyber threats.

Keywords: machine learning, cybersecurity, North American bulk power system.

\section{INTRODUCTION}

\subsection{Overview of Problem}

In 2017, the estimated cost of resolving a single cyberattack reached \$1.6 million dollars, taking 33 percent longer to resolve compared to five years before (Navy 2017). Many of these attacks begin by targeting the user, as users are typically the most vulnerable aspect of any system. By utilizing social engineering tactics such as phishing, spear phishing, and water holing, hackers easily deceive users into believing that they are a trusted party in an online interaction. From there, hackers gain their initial point of entry into their targeted system. Once initial access is gained, cyberattacks can spread across digital networks at an unprecedented rate leading to devastating impacts on government, corporations, and infrastructure. For everyday consumers alone, the annual global cost of cyberattacks was estimated to be $\$ 113$ billion dollars in 2017 (Navy 2017).

Cyberattacks drain both human and monetary resources in order to resolve impacts of an attack. With intrusions into critical U.S. infrastructure up 17 times over the past three years, more time will be spent defending against cyberthreats and resolving cyberattacks (Navy 2017). A well-timed attack could even. hamper the ability of the U.S. military to be highly deployable and prepared to dominate on the battlefield 
(Command 2015). With critical systems offline, the military would no longer be able to enable the Joint Force to gain the informational advantage, strike at long distance, and exercise global command and control (Defense 2018). As cyberspace becomes ubiquitous to operations, protecting these systems is becoming more crucial (Research 2014).

The WannaCry cyberattack, was a computer worm which impacted over 230,000 computers across over 150 countries in 2017 (Nakashima and Rucker 2017). In the first 24 hours alone, the attack infected 150,000-plus computers in those 150 countries (Navy 2017). The high impact of the attack can be attributed to the type of malware used, a computer worm. Computer worms function similarly to viruses with an ability to self-replicate across a digital network to reach more devices and are typically intended to destroy information in the form of data or files.

A more recent Russian led attack in 2018 known as NotPetya had a similar effect but used an alternative method of propagation known as a water holing (Nakashima 2018). In this case attackers targeted a trusted Ukrainian website that delivered updates for tax and accounting software. By targeting such a widely used website hackers created a watering hole to spread their attack that would reach a large audience and infect users' devices as they navigated the website. In this instance data was wiped from banks, energy firms, senior government officials and an airport. This demonstrates how even trusted websites and services can be at risk, leaving users vulnerable.

Consumers are not the only targets of cyberattacks. The Department of Defense (DoD) experiences 41 million scams, probes and attacks per month (Navy 2017). Government, military, and infrastructure systems are also susceptible to cyberattacks and experience many of the same vulnerabilities as they utilize commercial products and services. Back in 2015, the Office of Personnel Management (OPM) experienced a data break potentially compromising the personnel records of 21.5 million current and former federal employees (Navy 2017). Information from the attack could easily be used in future access-based attacks, where compromised passwords are exploited to gain access to a digital network.

These events, WannaCry, NotPetya, and the OPM data breach, are becoming known as high impact low frequency (HILF) events. However, the low frequency aspect is beginning to change as these events are becoming more common. Simultaneously, these events are also becoming more devastating as the methods and technology used are innovated and experience is gained. To make matters worse, a possibility exists that many of these attacks are only test runs that have yet to reach their full potential in ways of impact. By pairing alternative cyberattacks with such methods of propagation could lead to even more severe impacts on the affected system.

A major growing concern with many of these attacks is the possibility of targeting key infrastructure systems such as the bulk power system (BPS) or U.S. military's networks and systems. HILF events targeted at the BPS or U.S. military networks and systems could have profound consequences due to the many interdependencies on these networks and systems.

When it comes to the BPS, the North America Electric Reliability Corporation (NERC) has given Severe Events and their aftermath, the New Normal, special consideration. A Severe Event is an emergency situation so catastrophic that complete restoration of electric service is not possible (Force 2012). A coordinated physical attack and coordinated cyber-attack are HILF events also considered Severe Events. In the New Normal the BPS is operated at a reduced state of reliability and supply for months or possibly year (Force 2012).

\subsection{A Motivating Scenario}

A cyberattack can be initiated by simply identifying a soft target that is both highly susceptible and capable of delivering the most devastating impact. A microgrid may present a vulnerable target fitting the necessary criteria for a cyberattack. The unique disadvantage of each island being on an isolated (or islanded) power 
grid, emerge during the reconnaissance phase of the attack. At that point the main utility would also be identified as the sole electrical utility provider, giving hackers a more susceptible target than a main grid.

Hackers would perform reconnaissance on the utility provider and begin equipping themselves with a combination of authentic-looking email addresses and access to company employee contact email addresses. In order to successfully orchestrate a spear phishing attack, a variety of attempts tailored to different users may be necessary. Next, hackers would begin the intrusion phase embedding malware, such as spyware, into an authentic-looking email template personalized to each employee targeted. Launching subsequent attempts becomes easy; as the same targets can be used with an alternate template and sender address.

To complete the intrusion phase and move onto the exploration phase, only a single email of the countless attempts sent is required to successfully install the embedded spyware on the system. As spyware runs in the background, the intrusion is difficult to detect. Hackers collect credentials and other valuable information unnoticed. As more devices are compromised, hackers gain necessary information at a faster rate.

Once sufficient information is collected through the spyware and hackers gain access using compromised credentials; the attack begins to take action on the target. As the compromised log-in credentials are exploited, it is easy to bypass security measures since hackers appear as authorized users. With access to the island's power grid hackers can black out the entire island and remotely sabotage equipment including transformers, power lines, and substations rendering all solutions other than equipment replacement infeasible. A rippling effect could be noticed on all components, once generator reserves are depleted, unless equipped with a self-sustaining efficient microgrid.

As the blackout spreads across the microgrid's critical infrastructure; emergency services, medical services, and communications slow down until finally they are unavailable. Critical components will either blackout or operate with significantly depleted resources. The combination of the island being in a state of chaos and utility's weakened condition leave the island prone to physical attacks. With microgrid down, or resources nearly depleted, coordinating defenses, attacks, and counterattacks also becomes more difficult with limited communications and depleted information. The simplicity of this scenario demonstrates the need to fortify our cybersecurity systems against such attacks.

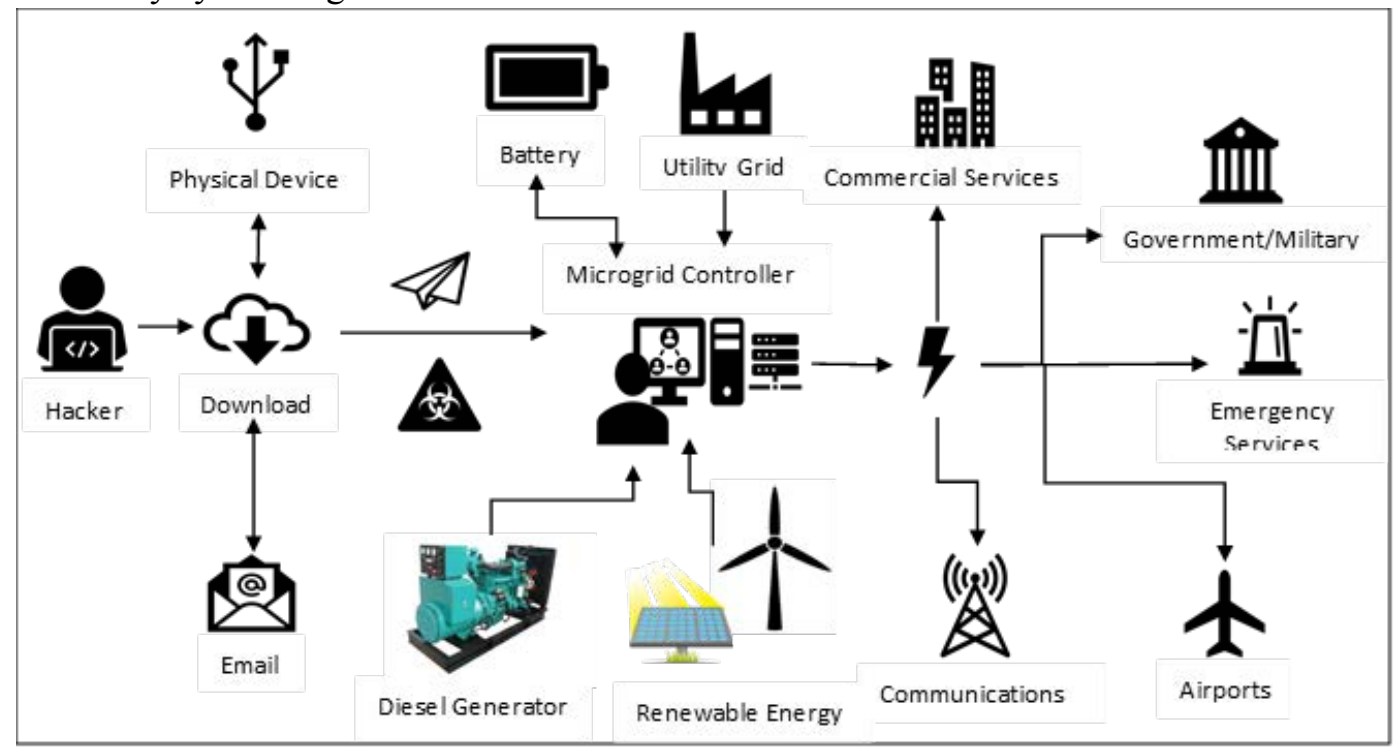

Figure 1: The interdependence of an electric grid. 


\section{CHALLENGES}

\subsection{System Challenges}

The North American BPS is divided into 8 regions and accounts for virtually all electricity supplied to the U.S., Canada, and a portion of Baja California Notre, Mexico (Force 2012). The sheer size and complexity of the BPS raise a multitude of challenges encountered on a regular basis. The complexity of the BPS arises from the interconnected nature of the system within itself and to other critical infrastructure (e.g., Communications, Information Technology, Hydroelectric, Coal, Oil, and Natural Gas), requiring system operators to constantly maintain situational awareness of the system condition and data from its connected neighbors. The system is also further subdivided into smaller regions for Reliability Coordinators (RC) to maintain reliable operations and Balancing Authorities (BA) to maintain load-generation balance within their footprints.

The composition of the BPS as a heterogeneous system, where both cyber and physical components are intertwined, creates further challenges, as both the operation and security of the BPS rely on a combination of personnel and digital systems (e.g., SCADA system, Automated Generation Control, and Energy Management System. Internally, the system is composed of substations, power plants, and the power supply all operated and controlled on a SCADA system by operators (e.g., BA, RC, Independent System Operators (ISO), and Regional Transmission Operators (RTO)). As the SCADA system allows for both remote operations and situational awareness of the system, it is vital to system operations. Externally, the BPS facilities must be secured by a combination of security equipment (e.g., surveillance, motion sensors, and access control) all monitored by security personnel. Coordinating and completing the competing objectives of operations and security simultaneously can be difficult with limited resources available, and sometimes require sacrificing one for the other. The heterogeneous nature of the system creates a wide variety of security threats including physical attacks, cyber-attacks and potentially a coordinated attack on both fronts.

Control centers are central to the operations of the BPS, acting as a hub to take in all the information from the system and provide operators with a space to come together to coordinate operations. Both a primary and back up facility are necessary in case one fails or is taken offline. Special design considerations must be considered when deciding where locate control centers. When considering a new location for the primary or alternate control centers, it is important to build the new facility a distance from the other which would avoid common risks such as natural disasters and hazards, as well as man-made concerns. However, the greater the distance between control centers the longer it would take to transition to the back up control center.

Certain limitations on both cyber and physical resources pose a constant challenge for the BPS. Resources must be efficiently delegated to maintain reliable operation. Personnel for maintenance and repairs, planning, logistics, and operations are all finite under the given budget for employing personnel. During emergencies, additional stress can be placed on personnel as workloads increase and overtime is necessary, ultimately increasing the risk of human error.

Both operational equipment and spare equipment are limited and may be located too far away to provide any immediate relief. In the event of an emergency, spare equipment for replacements and repairs can be critical to restore the system and hard to find when unavailable. Specialized equipment such as high voltage transformers, circuit breakers, turbines, phase shifters, and series capacitors often take a year or longer to procure and build (Force 2012). This requires planners to make certain design considerations such as stocking spare specialized equipment to be quickly accessible in case of an emergency. In some cases, old equipment or equipment not immediately essential can be cannibalized to provide parts for repairs. However, this can have long term consequences as the full system is restored and additional resources are needed. In the event of a national emergency, the BPS could also face additional limitations on fuel for backup generators and transportation as it competes with the need of national defense. 
As previously mentioned, following a Severe Event the BPS may be forced to operate at a New Normal for a prolonged period of time (i.e., months or possibly years). This unstudied state can bring about unforeseen challenges, placing a large burden on planners. In the operations end, equipment damage and resource limitations can force the BPS to be operated as a large number of electrically disconnected islands, reducing the stability and reliability inherent in the large interconnected BPS (Force 2012). Island operations are nothing new, as the BPS operates as four large islands known as Interconnections. Due to the BPS's interconnected nature, islands may comprise areas that fall under the authority of several different operating entities posing an additional jurisdiction challenge (Force 2012). The New Normal could require load shedding and rotating blackouts, which could result in electricity disruption to other critical infrastructurepotentially increasing the likelihood of errors (Force 2012).

Reliable operation of the BPS is highly dependent on other critical infrastructures. Certain infrastructures such as Transportation, Emergency Services, Oil/Gas, and Communications are critical to BPS operations. Transportation enables the movement of personnel and equipment, along with enabling Emergency Services to reach remote sites. Emergency Services (e.g., Police, EMT, and Fire) provide security to critical facilities and support during an emergency. Oil/Gas play an important role providing fuel for both transportation and backup generators to restart a plant from a black start. Finally, Communications play key roles enabling system operators to coordinate with each other, and other services to maintain reliable operations — such as emergency services.

Hydroelectric dams are a substantial generation resource in North for the BPS. Hydroelectric dams often provide critical black start services after a Severe Event; however, these facilities are limited due to the cost of standby equipment. In hydroelectric dams, backup generators must produce just enough power to open gates to allow the flow of water. Hydroelectric dams also serve other purposes intertwined in critical infrastructure such as controlling water flow for irrigation, navigation, and elevation (Force 2012).

Coal, oil, and natural gas act as another significant electricity generation fuel sources. Oil and Natural Gas also fuel backup generators and as fuel for service vehicles. According to the U.S. Energy Information Administration, coal currently accounts for almost half the U.S. electricity generation (Force 2012). The challenge is coal-fired generators are dependent upon frequent, in some cases daily, supply of coal from mine to power plant (Force 2012). Natural gas also faces challenges at pipeline compressor facilities as some are gas powered and others are electric powered, and a sustained BPS outage may cause gas pressure drops.

\subsection{Cyber Nature of Intended Target}

The vast size and complexity of the BPS requires a combination of human, machinery, and automation to ensure reliable operation. The supervisory control and data acquisition (SCADA) system is the backbone of the BPS cyber infrastructure as it helps maintain efficiency, process data for better decisions, and communicate system errors to mitigate downtime. SCADA plays a key role creating a human-machine interface (HMI) allowing operators to interact with equipment on site or remotely. The HMI also allows operators to monitor, gather, and process data in real time to understand and mitigate issues in the system. Real time data output allows operators to quickly trace the source of errors to mitigate downtime.

The basic SCADA architecture starts off with a collection of sensors gathering data on system operations. The sensors then feed the data to the programmable logic controllers (PLCs) or remote terminal units (RTUs). Both PLCs and RTUs are microcomputers that communicate with various components (e.g., factory machines, HMIs, sensors, and end devices) to then feed data into the SCADA system. Finally, the SCADA software process, distribute, and display the data to aid operators with analysis for better decisions regarding the system.

As the BPS evolved over time growing in size and complexity, it became infeasible for personnel to manually control and monitor equipment. With more facilities becoming necessary to sustain reliable 
operations, a capability for facilities to be remotely operated became imperative. SCADA posed a solution to these problems, then was followed up by automated system controls to meet the growing complexity. SCADA interfaces operators with various automated systems such as Automated Generation Control (AGC), Automatic Voltage Regulation (AVR), Energy Management System (EMS), and Generation Management System (GMS). Each system plays a key role ensuring reliable operation of the BPS with their ability to rapidly make decisions based on the status of the system, in a way an operator cannot keep up with.

Following a Severe Event, operation of automated systems may be unavailable or not practical to the situation. Without automated systems operation of the BPS faces challenges as operators struggle to keep up with system demands. Immediately following a Severe Event, the Immediate Automatic Response takes effect. The Immediate Automatic Response triggers automatic under-frequency load shedding and generator tripping may also occur as protective relays react to the transient voltages, frequency and power flows caused by the separation (Force 2012). Islands with small amounts of generation and load have less inertia and as such experience larger frequency swings, are harder to control, and more likely to collapse from subsequent generation loss than are the four existing Interconnections (Force 2012). Further complications are encountered with the increasing use of automatic schemes within the distribution system for "self-healing" or smart grids, due to the uncoordinated load restoration possibly increasing the risk of an island collapsing.

The Energy Management System (EMS) provides system operators with data and analyses to monitor and operate the transmission system, essentially offering of a "model" of the BPS. (Force 2012). The State Estimator then uses the model to calculate data points that are not physically metered, and also can help validate data or estimate missing data in the case of metering failures. The EMS also functions Security Analysis software providing sophisticated "What If" contingency analysis so operators can be prepared in the event that an element of the BPS, such as generating units or transmission lines, become unavailable.

The EMS also functions the AGC system, another vital component of BPS operation used to adjust the power output of multiple generators across different locations in response to changes in load. Power grids constantly require a close balance between generation and load, leading to a need for frequent adjustment to generator output. However, following the formation of an island, potentially due to a Severe Event, it may not be viable to have a centralized dispatch function based on bids and offers, so system operators will need to have an alternative means to dispatch (Force 2012). The AGC system may become unavailable due to limitations on the number of islands it can support, loss of frequency, or tie-line measurements.

The Generator Management System (GMS) is another important BPS system also playing into functioning the AGC. The GMS functions to provide power plant operators with data and analysis to monitor, control and operate multiple power plants to keep generator resources on schedule.

Finally, in recent years the Automatic Voltage Regulator (AVR) has become increasingly important to reliable operations as distributed generation with renewable resources create bi-directional power flows. The AVR functions to regulate voltage, by taking the fluctuate voltages and changing them into a constant voltage. The main reason for this is that variation in voltages damages power system equipment.

\subsection{Cyber Vulnerabilities of the Intended Target}

The cyber nature of the BPS opens a variety of possibilities for an attack on the system. With so much dependent on the ability to communicate, share data, and run automated control systems, the unavailability of even one aspect of the system could hamper reliable operations of the BPS. Through backdoors, cyberattacks, and user manipulation the possibilities for an attack are endless. Making matters worse, hackers continue to improve and refine these techniques creating new possibilities to be accounted for each day.

Control centers are a popular target for cyber-attacks acting as a nerve center for the entire system. From a cybersecurity perspective, both control centers could be significantly degraded if the primary and backup 
control centers are simultaneously exploited through the means by which entities keep the facilities synchronized (Force 2012). New recommendations to increase the separation between facilities mitigates risks associated with a physical attack or natural disaster, but still leaves the system vulnerable to cyberattacks. By not keeping the facilities synchronized and using different controls and hardware, the risk of common vulnerabilities between both control centers can be eliminated.

A majority of attacks on the BPS aim to gain unauthorized access to the system, while others block or deny access to the system or certain features from the intended users. Attacks attempting to gain unauthorized access to the system usually come in the form of backdoors, user manipulation, password attacks, or stolen credentials.

Backdoors are an exploit, typically built in during the design or manufacturing phase, that can later be used to gain unauthorized access to the system. In some cases, backdoors are simply an accident while others are part of a more elaborate plan to hijack the system or syphon data. A growing concern lately has been the use of commercial products or services sourced overseas due to the possibly of having built in backdoors.

User manipulation, stolen credentials, and password attacks have many commonalities and are typically used in conjunction with one another to gain unauthorized access to the system. User manipulation acts as one of the simplest and most effective means of transmitting an attack. Take, for instance, the Stuxnet attack where system users carried in a physical device, a USB drive, to an air gapped facility. After that, they connected it allowing them to infect the entire system. Air gapped systems, once thought to be impenetrable, even have vulnerabilities stemming from the user as the weakest link. Attacks such as Man in the Middle (MITM) and Drive-by Download also must employ an aspect of user manipulation to be successful.

On the other hand, Denial of Service (DOS) or Distributed Denial of Service (DDOS) attacks aim to disrupt or shutdown system operations. Unlike DOS, DDOS cannot be simply solved by blocking traffic from a single source since the traffic can be difficult to distinguish as multiple unique IP addresses are employed. Smurf attacks and SYN flood seems to be the most popular among denial of service attacks, where both send a large number of requests to an intended target to tie up system resources.

User targeted attacks not only steal credentials and data but will also attempt to install malware. Malware comes in a variety of forms including spyware, viruses, worms, trojans, ransomware, and rootkits. Each attack has unique aspects making it advantageous in certain situations, as determined by the attacker. The wide array of malware available further complicates the defenses against them.

\section{MODELING THE PROBLEM}

\subsection{Similar Research \& Concepts}

The preliminary element in addressing the problem of cyber-attacks is the detection of, and response to, anomalies in an area of the power grid. It is essential to establish a data collection method that will allow for quick response times. Other research surrounding the control of microgrids and distribution grids have made use of micro-PMU (Phasor Measurement Unit) for real-time analysis (Jamie et al. 2017). These collect data across the distribution system to allow for near instantaneous readings of the state of the system. The data consist of voltages and currents in different points on the system, and this can be useful in the identification of power outages or large surges that may be indicative of a cyber-attack. This information is then relayed to the control center, where operators can take proper actions against any detected anomalies.

The issue that arises with this collection system is the immense amount of data that is transmitted continuously. It is important to first establish a filtering method, so only very large or otherwise notable anomalies are made note of. This will exclude small spikes or drops in voltage that may be innocuous or indicative of an event other than a cyber-attack. It is also important to be able to instantly recognize an anomaly without the data needing to be sent to the control center first. Machine learning and deep learning 
can aid in this process by training a model with data from the power grid so that it learns the standard operations of the system. Then, the model will be able to detect anomalies that differ from the training data it has learned from. Research on deep learning with a power grid controller has shown promising results for anomaly detection, giving us a blueprint to apply the technique on a larger scale (He et al. 2019).

An inherent problem that exists with machine learning is the risk of manipulation from an attacker that can reduce the model's effectiveness. As hackers become more and more sophisticated, this is a particularly concerning vulnerability. For example, if the training data used by a machine learning algorithm is altered by an outside source, then the classification of inputs may be less accurate moving forward. Research on strengthening the robustness of algorithms through "stacking"-combining the predictions of different algorithms to form as input data for new ones - has shown this to be an effective method of countering this problem (Devine and Bastian 2019). Therefore, this cyber threat can potentially be mitigated through the combination of different machine learning models.

An important consideration when modeling a power grid is the type of data used - either persistent, or static, data or dynamic data. Dynamic data has the advantage of being more flexible than static data, allowing for analysis of the model under a wide variety of conditions. The ability to periodically update the data set is crucial for evaluating the response to different threats, and we believe it is more useful than static data in this particular model. Moreover, a Dynamic-Data-Driven Application System (DDDAS) can be incorporated as well, which allows for faster computation speed and higher accuracy of results.

\subsection{Creating the Model \& Proposed Solutions}

We propose that the combination of these methods will allow for an effective and adaptable system of cyber risk detection and assessment. The real time detection of anomalies can be accomplished through machine learning, allowing for response times and accuracy rates that will far outpace any human's capabilities. Using dynamic data to train the algorithm will also give an additional layer of versatility to the model — an important component when working with critical infrastructure with as much activity as this one.

The limitations present in these approaches are mostly due to the unpredictability and constant evolution of cyber-attacks. It is impossible to foresee and prepare for every form of attack when a large number of unknown threats exist. This is why the model emphasizes adaptability so the defender can form a response plan to a multitude of actual and theoretical threats. Despite this, we must acknowledge the reality that not every attack will be accounted for when simulating cyber threats.

Having established methods of improving cyber threat assessment, it is then important to decide how to model the power grid and the various threats it may face. For this, we elect to use simulation because of the versatility of designing different attacks and varying the parameters of the power grid. The main drawback of using simulation is the computing power needed for complex models. To alleviate this, the system will also be scaled down to analyze a specific substation of the entire power grid. This will help to produce detailed results that can then be extrapolated on a larger scale, without facing the limitations of computing power that would be an issue if the entire grid was modeled. The simulation platform to be used will be AnyLogic and the model will be agent-based, allowing for different behaviors and system dynamics. The model will consider a distribution network with coal-powered generators disseminating power to households and other consumers based on their demand. According to the U.S. Energy Information Administration (EIA), the average household consumed $28.9 \mathrm{kWh}$ per day, or about $1.2 \mathrm{kWh}$ every hour. (EIA 2019) However, such numbers can vary drastically from hour to hour and from consumer to consumer so this demand will be modeled stochastically. The consumers will request a certain amount of energy every hour according to an exponential distribution with rate $\lambda$ :

$$
\lambda=(1 / 1.2)=0.83 \text {. }
$$

The generators, along with sending power to consumers, will also periodically store power in a battery for future use. This power can then be sent back to the generators when the household demand necessitates it, 
and it will be distributed according to that demand. To model the risk present in a cyber-physical system, the attack on the power grid will consist of two prongs - a physical disruption to the infrastructure, such as extreme weather or military action, and a cyber-attack that targets the generators to halt their distribution of energy. This would essentially represent the worst-case scenario for the grid, where an already-weakened structure is then exploited further by a hacker. The attack speed and effectiveness will depend on the proficiency of the attacker - a parameter we can vary to model different scenarios.

In the initial model of the attack, there will be 3 generators servicing 15 consumers. This can be increased or decreased simply be adding or removing an address in the database of the simulation platform. The consumer or generator will be positioned at that specific address when running the model. To simulate the alternating current (AC) that is omnipresent in power distribution, the power will periodically reverse direction and flow from the consumers back to the generators. This will also aid in limiting the computational power needed to run the simulation because the agents that represent power can essentially be "reused" when they return to the generator. This will prevent constantly needing to produce new power agents, which would become an issue when the model is increased to a larger scale.

\subsection{Existing Threats to Industrial Control Systems}

Cyber-attacks compromise networks through various means including system updates, removable drives, public-facing web services, and email attachments (e.g. MS Office document). Once compromised, many attacks aimed at critical infrastructure will deploy a lightweight backdoor that does not require administrative privileges. The lightweight backdoor will then allow attackers to map the network and collect passwords to gain administrative privileges. With administrative privileges a proprietary backdoor is then opened allowing attackers to deploy additional software modules containing the payload. The software modules targeting industrial control system (ICS) components such as PLCs, SCADA, switches, and circuit breakers. To cover their tracks command and control (C\&C) servers used to dispatch modules are Tor relays hindering traffic analysis.

The software modules delivering the payload typically build off previous attacks such as EternalBlue and Mimikatz in the case of NotPetya, or GreyEnergy evolving out of BlackEnergy. In the case of NotPetya while not specifically aimed at infrastructure, the pandemic spread of the attack across networks crippled a variety of services. While NotPetya demanded a ransom, any payment was futile as the data was ultimately wiped a common occurrence when encountering ransomware. Moving back to attacks specifically aimed at critical infrastructure, the severity of such attacks has increased by orders of magnitude as they have become easily scalable due to the automated nature. The automation allows for attacks to be set off at a specific time or spread across a network without communication from the operator, in many cases leading to unintended collateral damage across connected networks. In the final stage of the attack a data wiper component is deployed in all mentioned attacks to cover the attackers track.

Table 1: Types of threats faced by ICS.

\begin{tabular}{|l|l|l|l|l|}
\hline $\begin{array}{l}\text { Type of } \\
\text { Attack }\end{array}$ & Motivating Scenario & Possible Impact Pathway & $\begin{array}{l}\text { Suspected } \\
\text { Advanced } \\
\text { Persistent } \\
\text { (APT) Group }\end{array}$ & $\begin{array}{l}\text { Severity } \\
(1-5)\end{array}$ \\
\hline Malware & GreyEnergy; & Compromise a public-facing & GreyEnergy & 5 \\
\hline
\end{tabular}




\begin{tabular}{|c|c|c|c|c|}
\hline & $\begin{array}{l}\text { BlackEnergy } \\
\text { predecessor, that deploys } \\
\text { GreyEnergy mini a } \\
\text { lightweight backdoor to } \\
\text { collect password and } \\
\text { gain administrative } \\
\text { privileges to deploy their } \\
\text { flagship backdoor to take } \\
\text { control of the network }\end{array}$ & $\begin{array}{l}\text { web service connected to the } \\
\text { internal network to sneak } \\
\text { inside the network, or spear } \\
\text { phishing emails containing } \\
\text { malicious attachments (ESET } \\
\text { 2019) }\end{array}$ & & \\
\hline $\begin{array}{l}\text { Ransomware; } \\
\text { Worm }\end{array}$ & $\begin{array}{l}\text { (Not)Petya; spread across } \\
\text { networks demanding } \\
\text { ransom for encrypted } \\
\text { files, but ultimately wipes } \\
\text { data }\end{array}$ & $\begin{array}{l}\text { Introduced to victim networks } \\
\text { via the update process to the } \\
\text { ME Doc tax software in } \\
\text { widespread use in the } \\
\text { Ukraine (Infosec 2017) }\end{array}$ & TeleBots & 4 \\
\hline $\begin{array}{l}\text { Malware; } \\
\text { Trojan }\end{array}$ & $\begin{array}{l}\text { Win32/Industroyer; } \\
\text { strongly suspected of } \\
\text { leading to power outages } \\
\text { in Ukraine in December } \\
2016\end{array}$ & $\begin{array}{l}\text { once Trojan is executed, a } \\
\text { backdoor is opened on the } \\
\text { compromised device } \\
\text { (Cherapanov 2017) }\end{array}$ & Unknown & 3 \\
\hline $\begin{array}{l}\text { Malware; } \\
\text { simple DDoS } \\
\text { trojan }\end{array}$ & $\begin{array}{l}\text { BlackEnergy; leading to } \\
\text { the December } 2015 \\
\text { blackout in the Ukraine } \\
\text { capital of Kiev }\end{array}$ & $\begin{array}{l}\text { spear-phishing emails } \\
\text { combined with the } \\
\text { exploitation of } \\
\text { MS Office documents } \\
\text { (Lipovsky and } \\
\text { Cherapanov 2014) }\end{array}$ & BlackEnergy & 2 \\
\hline Worm & $\begin{array}{l}\text { W32.Stuxnet; targets ICS } \\
\text { to sabotage gas pipeline } \\
\text { or power plant facilities } \\
\text { by reprogramming PLCs, } \\
\text { as seen in Iran }\end{array}$ & $\begin{array}{l}\text { introduced to the environment } \\
\text { on a removeable drive, as the } \\
\text { computers used to program } \\
\text { PLCs are typically } \\
\text { disconnected from networks } \\
\text { (Falliere, et al. 2011) }\end{array}$ & $\begin{array}{l}\text { United States } \\
\text { and Israel } \\
\text { governments } \\
\text { strongly } \\
\text { suspected }\end{array}$ & 1 \\
\hline
\end{tabular}

\section{CONCLUSION \& FUTURE PROSPECTS}

\subsection{Conclusion}

As cyber and physical system become more intertwined and commonplace, cyber-attacks pose an increasingly urgent threat to the safety and security of the developed world. This is particularly true for Industrial Control Systems like the North American BPS, with hundreds of millions of people depending on its reliable operation. To stay one step ahead of the constantly evolving cyber-attacks, a flexible model is needed for threat assessment and preparation - which we propose can be done effectively using a DDDAS-based simulation. These systems have shown positive results in other models requiring instantaneous decision making, and their application to cyber-threat management is very promising. Furthermore, using machine learning to recognize anomalies across the distribution grid and highlight those that could be indicative of a hack will be effective in quickly responding to cyber-threats.

In the simulation created in this research, a small segment of the power grid was modeled with a continuous flow of power from generator to consumer based on demand. It was then demonstrated how a severe weather event, which weakened the physical infrastructure, coupled with a targeted hack on the generators could disrupt this flow and knock the grid offline. This model allowed for a variety of parameter changes 
such as increasing the number of consumers or generators, altering the hacking proficiency, or varying the generation rate, among others. This versatility will prove useful in fitting the model to a wide range of situations and locations across the power grid, with its many uniquely different segments. Its implementation will help defender anticipate and prepare for the very real possibility of a cyber-attack.

\subsection{Future Prospects \& Limitations}

The next stages in the development of the simulation would be increasing the sophistication of the model to more specifically account for the different forms of cyber-attacks possible. In its current form, the model is indiscriminate about the type of attack performed, as it is considering a general malware infection that will knock the generators offline. This prevents the adaptability of the model to the various types of malware described in Table 1, which will produce different responses and effects in reality. Furthermore, we must also consider non-malware-based attacks such as a Denial of Service (DoS) or Man-In-The-Middle attack which could potentially disrupt the power grid as well. The concentration of this paper has been on malware due to recent real-life attacks, but for optimal cyber threat assessment it is necessary to have a model that can properly react to as many attacks as possible.

In the future, it would also be pertinent to include the effects on other critical infrastructure if the power grid is disrupted. This includes emergency services, communication, transportation and other essential functions that are reliant on a working electrical grid. Currently, the focus is on how consumers will be affected when the distribution network is taken offline, but the disruption of any other critical infrastructure will have a severe impact on the area as well. This could be modelled by adding agents to represent emergency services, which would be reliant on functional communication between consumers, their station and other emergency workers. Then, the disruption of the electric grid could impede the emergency workers from being able to effectively communicate with all of these sectors. This loss of functionality would produce negative impacts that could then be quantified in monetary terms to allow for objective assessment of the damages from a cyber-attack.

\section{REFERENCES}

Cherepanov, A. 2017 “WIN32/INDUSTROYER A New Threat for Industrial Control Systems”. ESET. www.welivesecurity.com/wp-content/uploads/2017/06/Win32_Industroyer.pdf

Command, U.S.A.R.D.a.E. 2015. "U.S. Army Research Development and Engineering Command Strategic Plan”. U.S. Army. https://www.army.mil/,

Defense, D. o. 2018. “Department of Defense Cyber Strategy”. Department of Defense. media.defense.gov.

Devine, S. M. and N. D. Bastian. 2019. "Intelligent Systems Design for Malware Classification Under Adversarial Conditions.” arxiv.org/abs/1907.03149.

EIA. 2019. “"U.S. Energy Information Administration - EIA - Independent Statistics and Analysis.” How Much Electricity Does an American Home Use?". EIA. www.eia.gov/tools/faqs/faq.php?id=97\&t=3.

ESET. 2019. “ESET Unmasks ‘GREYENERGY’ Cyber-Espionage Group.” ESET.

www.eset.com/int/greyenergy-exposed/.

Falliere, N., et al. 2011. “W32.Stuxnet Dossier”. Symantec. https://www.wired.com/images_blogs/threatlevel/2010/11/w32_stuxnet_dossier.pdf

Force, S.I.R.T. 2012. “Severe Impact Resilience: Considerations and Recommendations". North American Electric Reliability Corporation.

He, Z., et al. 2019. "Power-Grid Controller Anomaly Detection with Enhanced Temporal Deep Learning." arxiv.org/pdf/1806.06496.pdf. 
Jamei, M., et al. 2017. “Anomaly Detection Using Optimally-Placed $\mu$ PMU Sensors in Distribution Grids.” arxiv.org/pdf/1708.00118.pdf.

Lipovsky, R., and A. Cherepanov. 2014. "Back in BlackEnergy: 2014 Targeted Attacks in the Ukraine and Poland." ESET. www.virusbulletin.com/conference/vb2014/abstracts/back-blackenergy2014targeted-attacks-ukraine-and-poland.

Nakashima, E. 2018. "Russian military was behind 'NotPetya' cyberattack in Ukraine, CIA concludes." Retrieved March 6 2019, from https://www.washingtonpost.com/world/nationalsecurity/russianmilitary-was-behind-notpetya-cyberattack-in-ukraine-ciaconcludes/2018/01/12/048d8506-f7ca-11e7b34ab85626af34ef_story.html?utm_term=.baea25c94d61.

Nakashima, E. and P. Rucker. 2017. "U.S. declares North Korea carried out massive WannaCry cyberattack." from https://www.washing-tonpost.com/world/national-security/us-set-to-declarenorthkorea-carried-out-massive-wannacrycyber-attack/2017/12/18/509deb1c-e446-11e7a65d1ac0fd7f097e_story.html?utm_term=.a708b558a5d9.

Nakashima, E. and P. Rucker 2017. "U.S. declares North Korea carried out massive WannaCry cyberattack." https://www.washingtonpost.com/world/national-security/us-set-to-declare-northkoreacarried-out-massive-wannacry-cyber-attack/2017/12/18/509deb1c-e446-11e7a65d1ac0fd7f097e_story.html?utm_term=.a708b558a5d9.

Navy, U. S. 2017. "An information graphic depicting the dangers of cyber-attacks." https://www.navy.mil/submit/display.asp?story_id=102685, U.S. Navy.

Infosec. 2017. "NotPetya, Complex Attacks, and the Fog of War." Infosec.engineering. infosec.engineering/notpetya-complex-attacks-and-the-fog-of-war/

Research, A. F. O. o. S. 2014. "Air Force Office of Scientific Research 2014 Technical Strategic Plan." https://www.wpafb.af.mil/afrl/afosr/, Air Force Office of Scientific Research.

\section{AUTHOR BIOGRAPHIES}

MICHAEL MESHAM is an undergraduate student in the Engineering Technology Department at Drexel University. Before studying at Drexel University, he studied in the Department of Industrial Engineering at the University of Miami. His research interests include cybersecurity, machine learning, and simulation. His email ismm5243@drexel.edu.

MAHMOUD FAHMY is an undergraduate student at the University of Miami pursuing a joint B.S/M.S degree in Industrial Engineering, with a minor in Economics. His research interests include simulation and optimization and their application in distribution systems. His email address is mxf898@miami.edu.

NURCIN CELIK is an Associate Professor and the Director of Simulation and Optimization Research Laboratory in the Department of Industrial Engineering at the University of Miami. She received her M.S. and Ph.D. degrees in Systems and Industrial Engineering. Her research interests include architectural design and application of dynamic data-driven adaptive simulations for distributed systems including power and cyber systems. Her email address is celik@miami.edu. 